\title{
PAMELA: A Satellite Experiment for Antiparticles Measurement in Cosmic Rays
}

Massimo Bongi, O. Adriani, M. Ambriola, A. Bakaldin, G. C. Barbarino, A. Basili, G. Bazilevskaja, R. Bellotti, R. Bencardino, M. Boezio, E. A. Bogomolov, L. Bonechi, L. Bongiorno, V. Bonvicini, M. Boscherini, F. S. Cafagna, D. Campana, P. Carlson, M. Casolino, G. Castellini, M. Circella, C. N. De Marzo, M. P. De Pascale, G. Furano, A. M. Galper, N. Giglietto, A. Grigorjeva, S. V. Koldashov, M. G. Korotkov, S. Y. Krut'kov, J. Lund, J. Lundquist, A. Menicucci, W. Menn, V. V. Mikhailov, M. Minori, N. Mirizzi, J. W. Mitchell, E. Mocchiutti, A. Morselli, R. Mukhametshin, S. Orsi, G. Osteria, P. Papini, M. Pearce, P. Picozza, M. Ricci, S. B. Ricciarini, M. Romita, G. Rossi, S. Russo, P. Schiavon, M. Simon, R. Sparvoli, P. Spillantini, P. Spinelli, S. J. Stochaj, Y. Stozhkov, S. Straulino, R. E. Streitmatter, F. Taccetti, A. Vacchi, E. Vannuccini, G. I. Vasilyev, S. A. Voronov, R. Wischnewski, Y. Yurkin, G. Zampa, and N. Zampa

\begin{abstract}
PAMELA is a satellite-borne experiment that will study the antiproton and positron fluxes in cosmic rays in a wide range of energy (from $80 \mathrm{MeV}$ up to $190 \mathrm{GeV}$ for antiprotons and from $50 \mathrm{MeV}$ up to $270 \mathrm{GeV}$ for positrons) and with high statistics, and that will measure the antihelium/helium ratio with a sensitivity of the order of $10^{-8}$. The detector will fly on-board a polar orbiting Resurs DK1 satellite, which will be launched into space by a Soyuz rocket in 2004 from Baikonur cosmodrome in Kazakhstan, for a 3-year-long mission. Particle identification and
\end{abstract}

Manuscript received November 21, 2003; revised March 22, 2004.

M. Bongi is with INFN-Sezione di Firenze, 1-50019 Sesto Fiorentino (FI), Italy (e-mail: bongi@fi.infn.it).

O. Adriani, L. Bonechi, P. Papini, S. B. Ricciarini, P. Spillantini, S. Straulino, F. Taccetti, and E. Vannuccini are with Università di Firenze and INFN, 1-50019 Sesto Fiorentino, Italy.

M. Ambriola, R. Bellotti, F. S. Cafagna, M. Circella, C. N. De Marzo, N. Giglietto, N. Mirizzi, M. Romita, and P. Spinelli are with Università di Bari and INFN, Bari 70426, Italy.

A. Bakaldin, A. M. Galper, S. V. Koldashov, M. G. Korotkov, V. V. Mikhailov, S. A. Voronov, and Y. Yurkin are with Moscow Engineering and Physics Institute, Moscow 115409, Russia.

G. C. Barbarino, D. Campana, G. Osteria, G. Rossi, and S. Russo are with Dipartamento di Scienze Fisiche, Università "Federico II" and INFN, Napoli 80126, Italy.

A. Basili, R. Bencardino, L. Bongiorno, M. Casolino, M. P. De Pascale, G. Furano, A. Menicucci, M. Minori, A. Morselli, P. Picozza, R. Sparvoli, and R. Wischnewski are with Università "Tor Vergata" and INFN, Roma-Tor Vergata 00133, Italy.

G. Bazilevskaja, A. Grigorjeva, R. Mukhametshin, and Y. Stozhkov are with Lebedev Physical Institute, Moscow 119 991, Russia.

M. Boezio, V. Bonvicini, P. Schiavon, A. Vacchi, G. Zampa, and N. Zampa are with Università di Trieste and INFN, Trieste 34127, Italy.

E. A. Bogomolov, G. I. Vasilyev, and S. Y. Krut'kov are with Ioffe PhysicoTechnical Institute, St. Petersburg 194 021, Russia.

M. Boscherini, W. Menn, and M. Simon are with Universitaet-GH Siegen, FB Physik, Siegen 57068, Germany.

P. Carlson, J. Lund, S. Orsi, and M. Pearce are with the Royal Institute of Technology, Stockholm 10691, Sweden.

G. Castellini is with Istituto di Fisica Applicata "N. Carrara, " CNR, Firenze 50127, Italy.

J. W. Mitchell and R. E. Streitmatter are with NASA Goddard Space Flight Center, Greenbelt, MD 20771 USA.

E. Mocchiutti was with Università di Trieste and INFN, Trieste, Italy. He is now with the Royal Institute of Technology, Stockholm 10691, Sweden.

J. Lundquist was with the Royal Institute of Technology, Stockholm, Sweden.

He is now with Università di Trieste and INFN, Trieste 34127, Italy

M. Ricci is with Laboratori Nazionali INFN, Frascati 00044, Italy.

S. J. Stochaj is with Particle Astrophysics Laboratory, NMSU, Las Cruces,

NM 88003-8001 USA.

Digital Object Identifier 10.1109/TNS.2004.829504 energy measurements are performed in the PAMELA apparatus using the following subdetectors: a magnetic spectrometer made up of a permanent magnet equipped with double-sided microstrip silicon detectors, an electromagnetic imaging calorimeter composed of layers of tungsten absorber and silicon detectors planes, a transition radiation detector made of straw tubes interleaved with carbon fiber radiators, a plastic scintillator time-of-flight and trigger system, a set of anticounter plastic scintillator detectors, and a neutron detector. The features of the detectors and the main results obtained in beam test sessions are presented.

Index Terms-Antimatter, cosmic rays, satellite experiment.

\section{INTRODUCTION}

$\mathbf{P}$ AMELA (a Payload for Antimatter Matter Exploration and Light-nuclei Astrophysics) [1] is a satellite-borne experiment that has been designed to study charged particles in cosmic rays. The detector will be mounted in a pressurized vessel attached to a Russian Earth-observation satellite, the Resurs DK1, which will be launched into space by a Soyuz rocket in 2004 from Baikonur cosmodrome in Kazakhstan. The orbit will be elliptical and semi-polar, with an inclination of $70.4^{\circ}$ and an altitude varying between 350 and $600 \mathrm{~km}$.

During its at least 3-year-long mission, PAMELA will measure with high precision the energy spectrum and the composition of the cosmic radiation, in particular looking for its antimatter component. It will extend the currently explored region of the antiprotons and positrons spectrum both toward higher and lower energies. The characteristics of the orbit will grant PAMELA sensitivity to the low energy region because of the lower Earth's geomagnetic cutoff near the poles.

\section{SCIENTIFIC OBJECTIVES}

The primary objective of the PAMELA experiment is to detect and identify antiprotons and positrons in cosmic radiation, measuring their spectrum in a wide energy range $(80 \mathrm{MeV}-190$ $\mathrm{GeV}$ for antiprotons, $50 \mathrm{MeV}-270 \mathrm{GeV}$ for positrons) with statistics never reached before $\left(\sim 10^{4}\right.$ and $\sim 10^{5}$ particles/year, respectively).

Almost all data available so far about cosmic antiprotons and positrons have been obtained using detectors in balloon-borne 


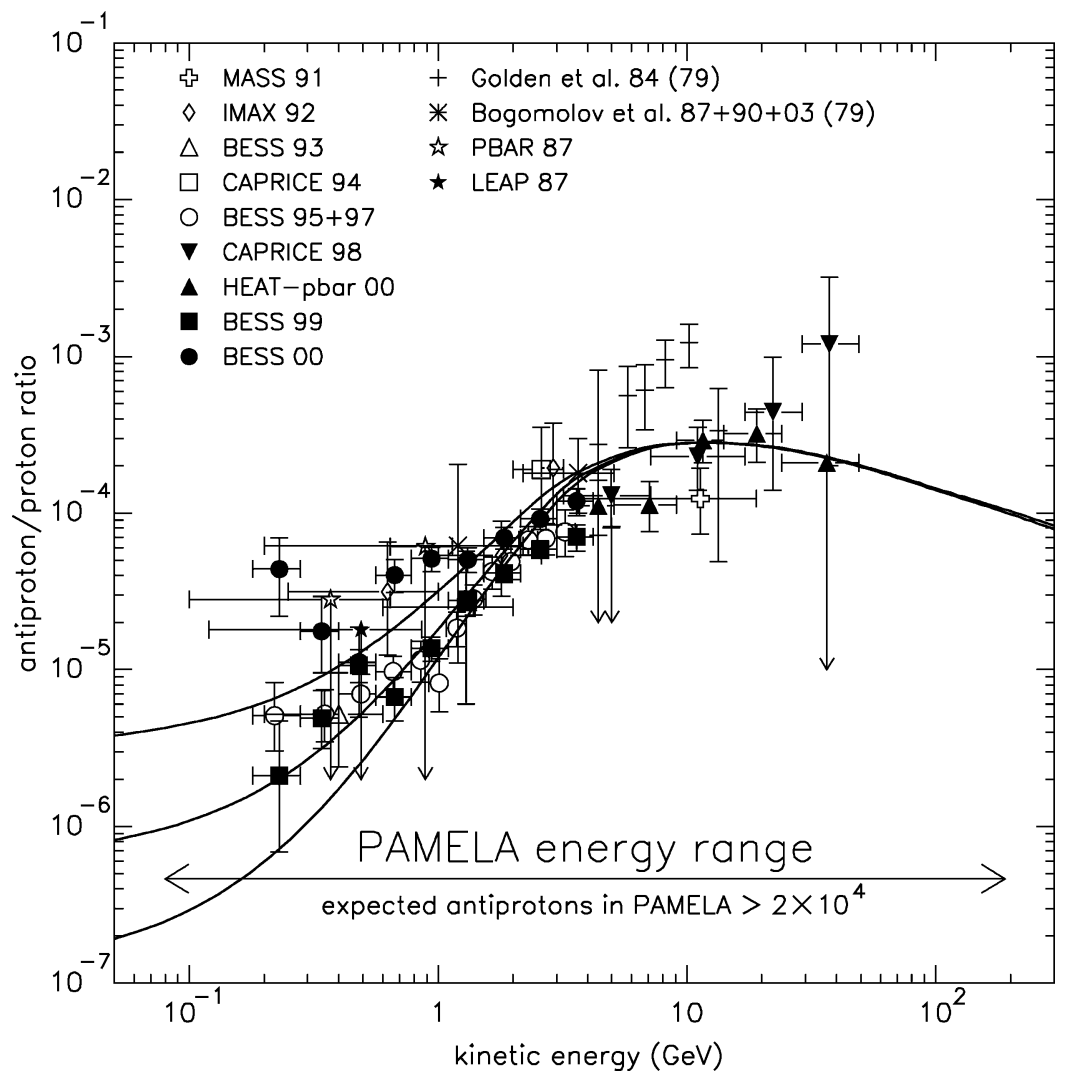

Fig. 1. Antiproton-to-proton ratio at the top of the atmosphere. PAMELA expected energy range is shown. References are: Golden et al. [7], [8], Bogomolov et al. [9] and references therein, LEAP 87 [10], PBAR 87 [11], MASS 91 [12], IMAX 92 [13], BESS 93 [14], CAPRICE 94 [15], BESS 95+97 [16], CAPRICE 98 [17], HEAT-pbar 00 [18], and BESS 99 and 00 [19]. Lines represent theoretical calculations based on a purely secondary production of antiprotons [20].

experiments. The short data-taking time (approximately $24 \mathrm{~h}$ ) and the presence of a residual amount of the Earth's atmosphere above the detecting apparatus at altitudes that a balloon can reach (from $\sim 10 \mathrm{~g} / \mathrm{cm}^{2}$ around $30 \mathrm{~km}$, to $\sim 3 \mathrm{~g} / \mathrm{cm}^{2}$ around $40 \mathrm{~km}$ ) are the main limits of such kind of measurements.

Figs. 1 and 2 summarize the current situation in antiproton and positron measurements in cosmic rays. A satellite-borne experiment such as PAMELA will be able to do more precise measurements and cover a wider range of energy, thanks to the lack of atmospheric overburden and the longer data-taking time. This will allow us to discriminate among competing models of antimatter production in our galaxy or to identify primary sources of cosmic antimatter.

The PAMELA experiment will also look for light antinuclei in cosmic rays, measuring the $\overline{\mathrm{He}} / \mathrm{He}$ ratio with a sensitivity $\sim 10^{-8}$, and it will study ordinary matter cosmic rays as well, detecting protons, electron, and light nuclei up to $\mathrm{Z}=6$.

Table I summarizes the expected particle samples after three years of operation of the detector.

\section{PAMELA SUBDETECTORS}

PAMELA (see Fig. 3) is built around a permanent magnetic spectrometer equipped with a silicon tracking system, which is used to measure the momentum, the sign, and the absolute charge of particles passing through the cavity of the magnet. An anticounter system (AC) surrounds the magnet and allows rejection of particles which do not pass cleanly through the acceptance window of the tracker. An electromagnetic calorimeter
TABLE I

EXPECTED PARTICLE SAMPLES AFTER a 3-Year PAMELA MisSION

\begin{tabular}{l|c|c}
\hline Particle & Number (3 years) & Energy Range \\
\hline \hline Protons & $3 \times 10^{8}$ & $80 \mathrm{MeV}-700 \mathrm{GeV}$ \\
Antiprotons & $>3 \times 10^{4}$ & $80 \mathrm{MeV}-190 \mathrm{GeV}$ \\
Electrons & $6 \times 10^{6}$ & $50 \mathrm{MeV}-2 \mathrm{TeV}$ \\
Positrons & $>3 \times 10^{5}$ & $50 \mathrm{MeV}-270 \mathrm{GeV}$ \\
$\mathrm{He}$ & $4 \times 10^{7}$ & $80 \mathrm{MeV} / \mathrm{n}-700 \mathrm{GeV} / \mathrm{n}$ \\
$\mathrm{Be}$ & $4 \times 10^{4}$ & $80 \mathrm{MeV} / \mathrm{n}-700 \mathrm{GeV} / \mathrm{n}$ \\
$\mathrm{C}$ & $5 \times 10^{5}$ & $80 \mathrm{MeV} / \mathrm{n}-700 \mathrm{GeV} / \mathrm{n}$ \\
$\overline{\mathrm{He}} /$ He limit at $90 \%$ C.L. & $7 \times 10^{-8}$ & $80 \mathrm{MeV} / \mathrm{n}-30 \mathrm{GeV} / \mathrm{n}$ \\
\hline
\end{tabular}

measures the energy of electrons and positrons and it is used for particle identification through the topological discrimination of the shower. In this task it is complemented at energies below $1 \mathrm{GeV}$ by time-of-flight (ToF) measurements made by a scintillator system, which also provides the primary experimental trigger, and at energies above $1 \mathrm{GeV}$ by a transition radiation detector (TRD), which makes particle identification through threshold velocity measurements. A bottom scintillator and a neutron detector placed below the apparatus will provide additional information which will help in distinguishing electromagnetic and hadronic showers in the calorimeter.

The detector is approximately $130 \mathrm{~cm}$ high, its mass is about $450 \mathrm{~kg}$, and the power consumption is $350 \mathrm{~W}$.

In this section, a more detailed overview of each PAMELA subdetector and the main results from beam test sessions are given. 


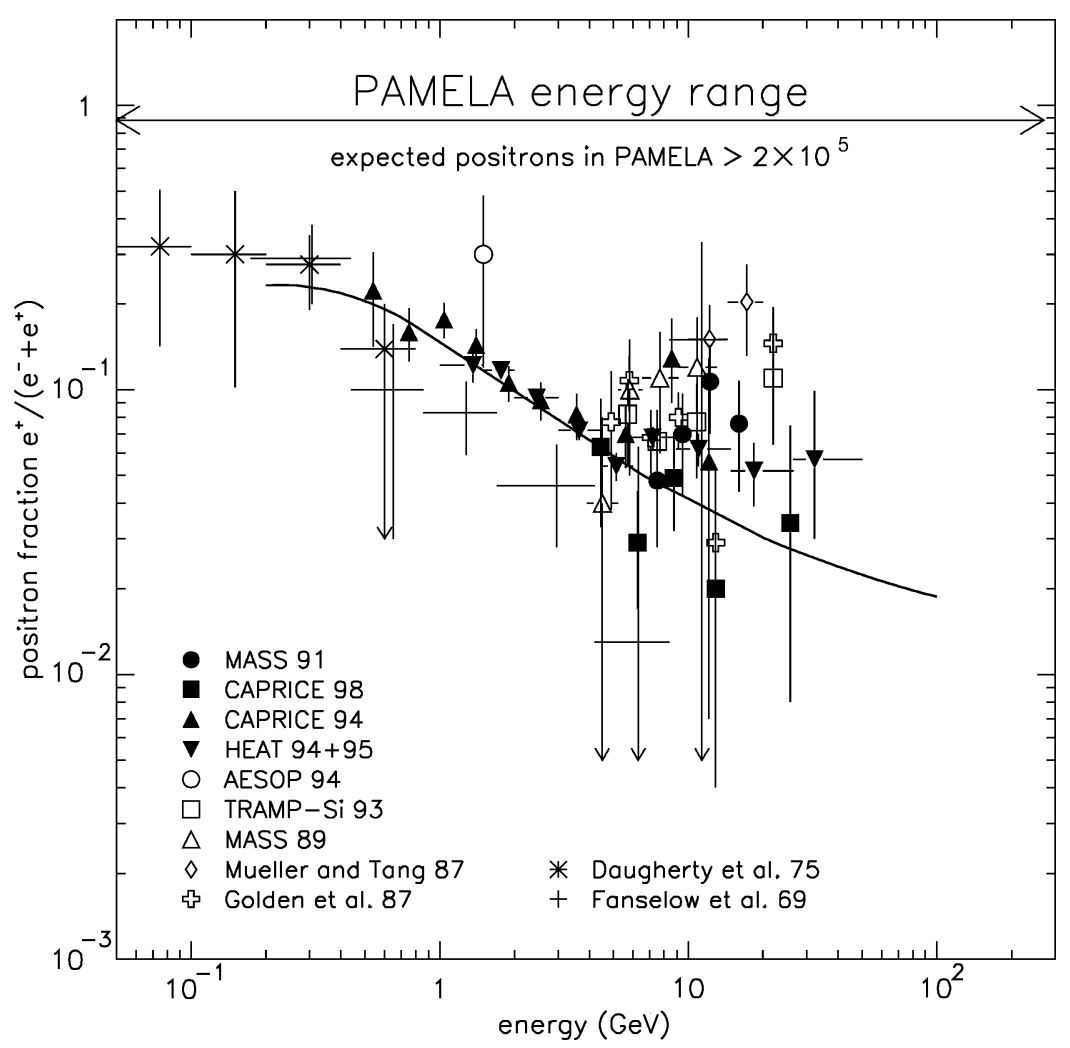

Fig. 2. Positron fraction at the top of the atmosphere. PAMELA expected energy range is shown. References are: Fanselow et al. [21], Daugherty et al. [22], Golden et al. [23], Mueller and Tang [24], MASS 89 [25], TRAMP-Si 93 [26], AESOP 94 [27], HEAT 94+95 [28], CAPRICE 94 [29], CAPRICE 98 [30], and MASS 91 [31]. The line represents a theoretical calculation based on a purely secondary production of positrons [32].

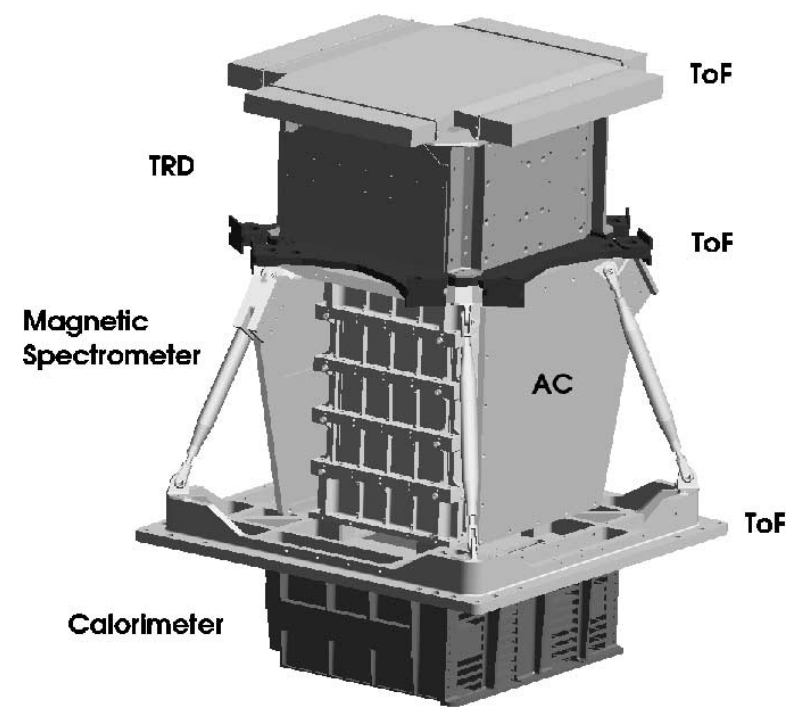

Fig. 3. Schematic view of the PAMELA detector, in which the mutual positions of the subsystems are shown.

\section{A. Magnetic Spectrometer}

The central part of PAMELA apparatus is a magnetic spectrometer [2] consisting of a permanent magnet and a silicon tracker.

The magnet is made up of five modules of a sintered $\mathrm{Nd}-\mathrm{Fe}-\mathrm{B}$ alloy with a residual magnetic induction of about 1.3 $\mathrm{T}$. The tracking cavity is $445 \mathrm{~mm}$ tall and has a cross section of
$132 \mathrm{~mm} \times 162 \mathrm{~mm}$, which gives $20.5 \mathrm{~cm}^{2} \mathrm{sr}$ as the geometric factor of the detector. The particular magnetic configuration permits a nearly uniform magnetic field inside the spectrometer cavity of about $0.4 \mathrm{~T}$.

The tracking system is composed of six detecting planes interleaved with the magnetic modules. Each plane is made up of six double-sided microstrip silicon detectors, $300 \mu \mathrm{m}$ thick. The strip pitch is $25 \mu \mathrm{m}$ for the junction side (which is used to measure the coordinate in the bending plane of the particles inside the magnetic cavity) and one strip out of two is connected to the readout electronics. On the ohmic side the pitch is $67 \mu \mathrm{m}$, strips are orthogonally implanted with respect to the previous ones, and a double metal layer is used in order to have the readout electronics on the same sensor edge for both sides. This configuration gives a total of 6144 readout channels for each of the six planes. The charge produced within silicon by ionizing particles is collected by the strips and used to reconstruct the impact points on the spectrometer's planes. In order to reduce the amount of data to be transmitted from the satellite a compression algorithm has been developed. The compression factor that has been obtained is about $95 \%$, with no degradation of the detector response.

Data acquired in beam test sessions allowed a measure of the spatial resolution of the silicon detectors (see Fig. 4). The result that has been obtained is about $3 \mu \mathrm{m}$ for the particle bending direction and about $11 \mu \mathrm{m}$ for the orthogonal coordinate (Gaussian fit). The resulting maximum detectable rigidity for the spectrometer exceeds $740 \mathrm{GV} / \mathrm{c}$. 


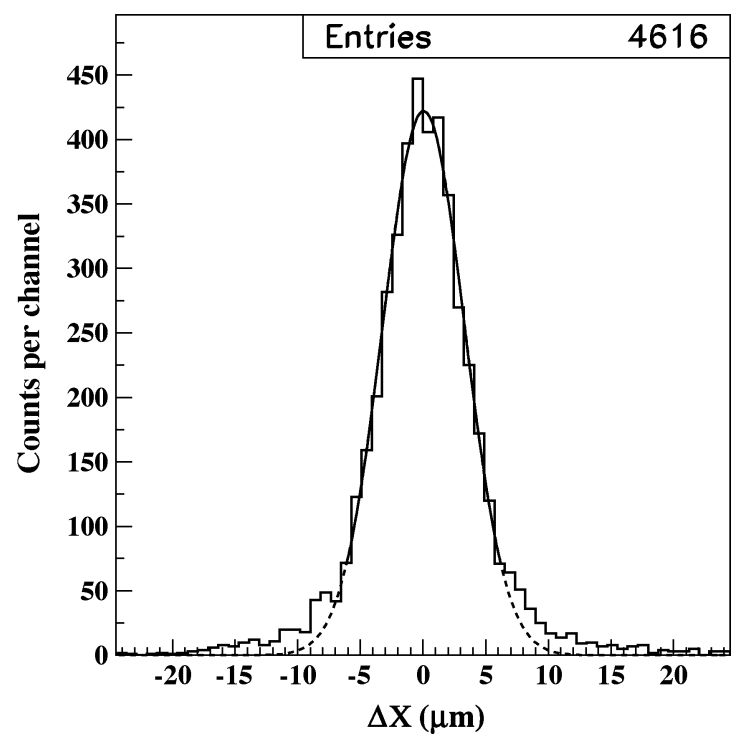

Fig. 4. Spatial resolution in the bending view for the tracker silicon detectors.

\section{B. Anticounter System}

The anticounter system [3] consists of four lateral detectors covering the sides of the tracker and one top detector above the tracker. Each detector is made from a sheet of plastic scintillator read out by multiple compact photomultiplier tubes, equipped with a LED based system for in-flight checks.

The efficiency for detecting minimum ionizing particles has been measured using cosmic ray muons and it has been found to exceed $99.9 \%$ per detector, thus permitting us to exclude from offline analysis particles which do not pass within the geometrical acceptance of the tracking system.

\section{Electromagnetic Imaging Calorimeter}

The electromagnetic calorimeter [4] is made from 44 planes of single-sided silicon detectors interleaved with 22 layers of tungsten absorbers. The detecting planes are $380 \mu \mathrm{m}$ thick and segmented in $2.4 \mathrm{~mm}$ strips, while the absorber layers are 2.6 $\mathrm{mm}$ thick. The total depth of the calorimeter is about 16.3 radiation lengths and about 0.6 interaction length.

The strips are arranged in a $\mathrm{Si}-\mathrm{x} / \mathrm{W} / \mathrm{Si}-\mathrm{y}$ geometry in order to allow topological reconstruction of the shower. It is thus possible to distinguish electrons and positrons from hadrons or noninteracting particles, obtaining a rejection factor of better than $10^{4}$ between protons (antiprotons) and positrons (electrons) at $95 \%$ retaining efficiency. The achieved energy resolution in beam test sessions is about $5.5 \%$ in the range 20-200 GeV, as shown in Fig. 5.

The calorimeter can also be operated in self-trigger mode: this hardware feature allows the stand-alone detection of $\mathrm{e}^{-}$with an increased acceptance of $470 \mathrm{~cm}^{2}$ sr up to an energy of about 2 TeV. Fig. 5 shows that an energy resolution of about $12 \%$ has been obtained in the range $200-700 \mathrm{GeV}$ : the rising of $\sigma(\mathrm{E}) / \mathrm{E}$ for higher energies is due to the incomplete shower containment at these energies.

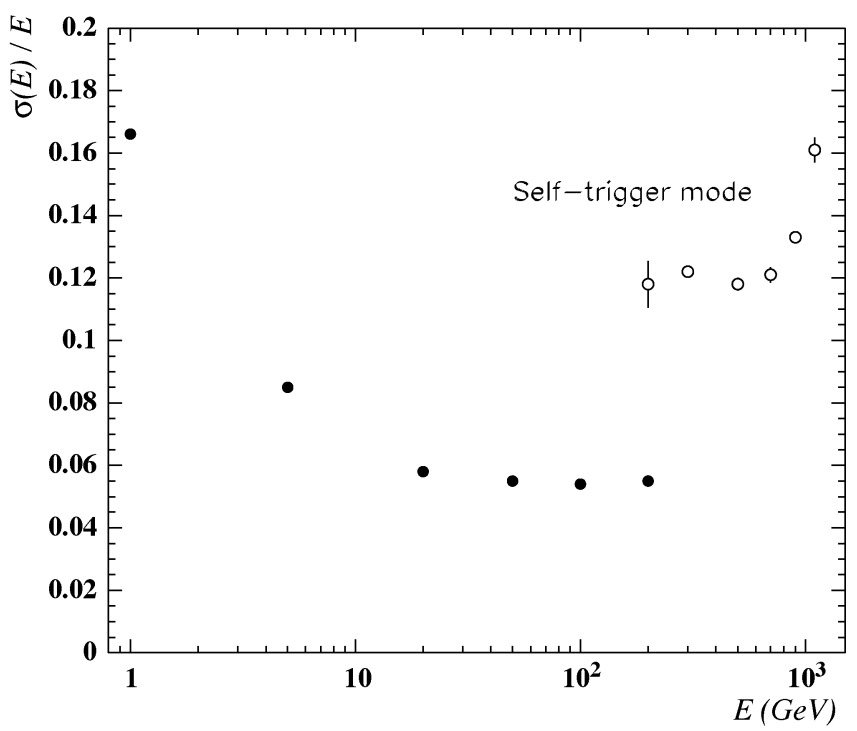

Fig. 5. Energy resolution of the electromagnetic calorimeter as a function of energy for electrons and positrons. The filled dots are for normal operation, while the open dots are for self-triggering mode.

\section{Time-of-Flight (ToF) System}

The ToF system [5] is composed of six layers of segmented plastic scintillators arranged in three planes and read out by photomultiplier tubes. The three planes are located above the TRD, between the TRD and the spectrometer, and between the spectrometer and the calorimeter (see Fig. 3).

The ToF provides a fast signal for triggering data acquisition in the other subdetectors, determines the absolute value of the charge $\mathrm{Z}$ of incident particles through multiple measurements of the energy loss $d E / d x$ in the planes, and finally measures the flight time of particles crossing its planes. Once this information is integrated with the measurement of the trajectory length done by the tracking system, the velocity of the particle can be calculated. This feature also enables the rejection of albedo particles.

An evaluation of the timing resolution of the planes has been performed comparing the impact point reconstruction done by the scintillator with the impact point obtained by an external drift chamber. Fig. 6 shows the distribution of the difference (converted to a time) between the impact point reconstructed by the $\mathrm{ToF}$ and the one obtained by the drift chamber, after corrections for time walk have been applied. The resulting timing resolution allows for a $3 \sigma$ separation between protons (antiprotons) and positrons (electrons) up to about $1.5 \mathrm{GeV}$.

\section{E. Transition Radiation Detector}

PAMELA TRD [6] is a detector made up of 10 carbon-fiber radiator layers interleaved with 9 planes of $\mathrm{Xe}-\mathrm{CO}_{2}$ straw tubes working in a proportional regime at a $1400 \mathrm{~V}$ voltage, arranged in a truncated pyramid shape. A single straw tube is $280 \mathrm{~mm}$ in length and $4 \mathrm{~mm}$ in diameter. It is made of a copper-clad Kapton foil, $30 \mu \mathrm{m}$ thick, with a tungsten anode wire, $25 \mu \mathrm{m}$ in diameter, stretched up to a $60 \mathrm{~g}$ tension, on the inside.

Relativistic particles crossing boundaries of materials with different dielectric constants (the carbon-fiber radiator layers interfaces) will emit transition radiation in the X-ray range, which is then detected by the gas detectors. For a given momentum, 


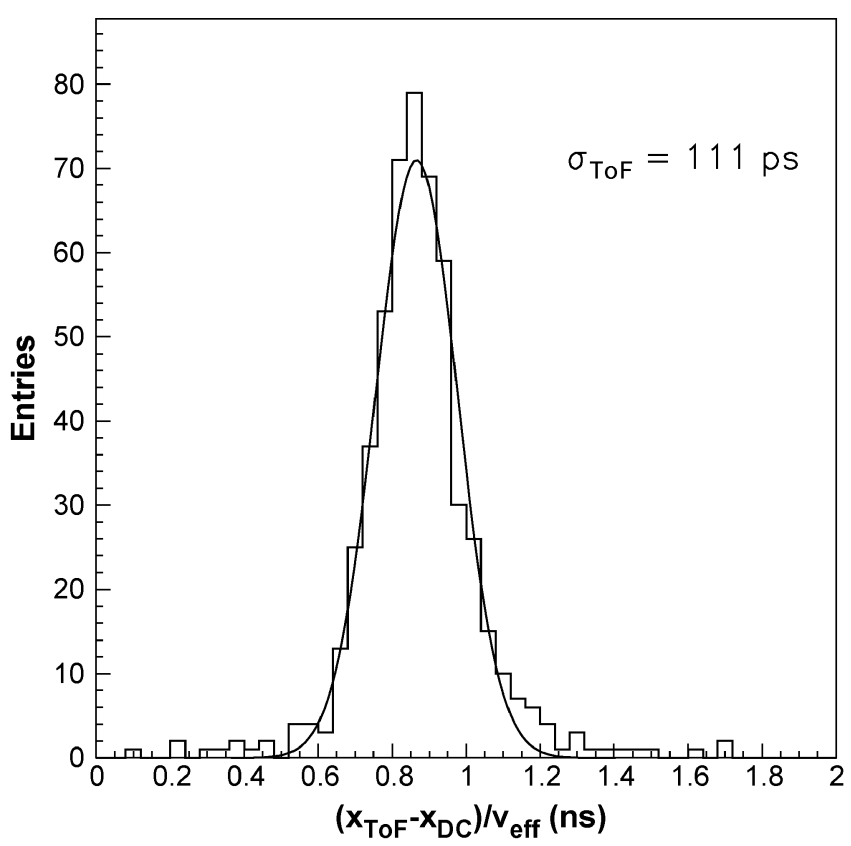

Fig. 6. Timing resolution for a ToF paddle, after correcting for time walk.

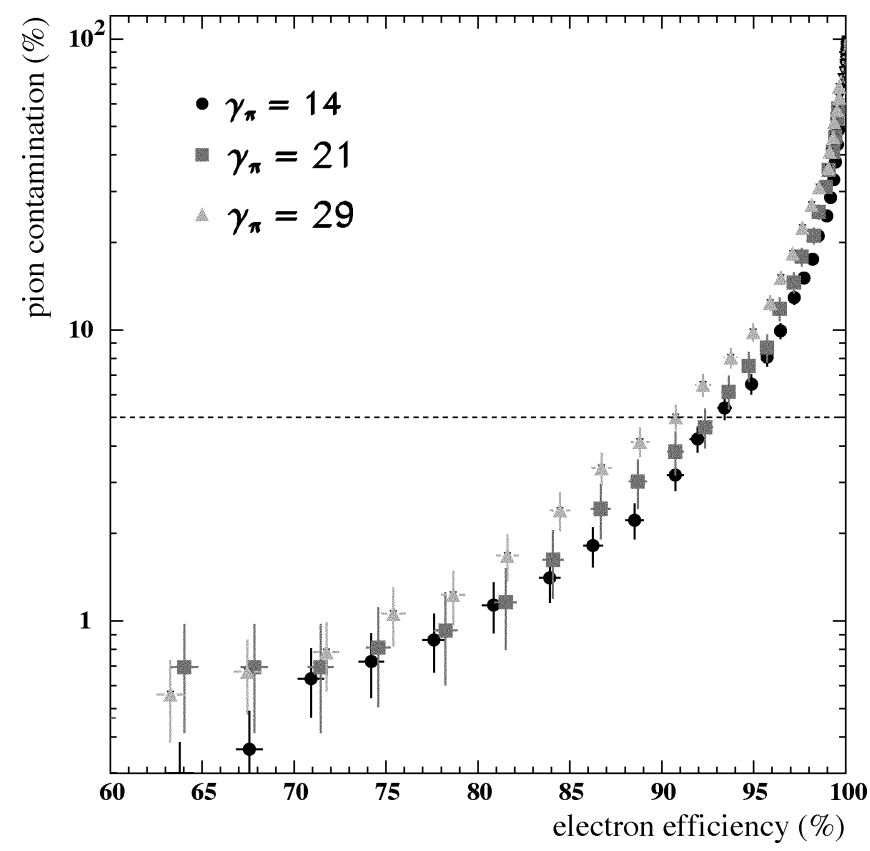

Fig. 7. Pion contamination versus electron discrimination efficiency at different Lorentz factors for the transition radiation detector.

the TRD allows a lepton-hadron separation through threshold velocity measurement. A discrimination efficiency of $90 \%$ for radiating particles with a contamination at a level of 5\% from nonradiating particles has been obtained in the PAMELA range of investigation (see Fig. 7).

\section{F. Bottom Scintillator and Neutron Detector}

A plastic scintillator plane located under the calorimeter detects particles escaping from it: this bottom scintillator is used to provide an additional trigger for high energy $(>100 \mathrm{GeV})$ electrons. It also triggers the neutron detector housed below it.
The purpose of this ${ }^{3} \mathrm{He}$ neutron detector is to extend the electron-proton discrimination capabilities of the PAMELA apparatus up to $10^{11}-10^{13} \mathrm{eV}$. A large neutron component will accompany high energy hadronic showers developed in the calorimeter.

\section{CONCLUSION}

In preparation to the space mission, extensive space qualification tests on PAMELA detectors, electronics, and mechanical structures have been developed and are still in progress. During 2000 and 2001, the mass and thermal model and the engineering model of PAMELA subdetectors were qualified for space flight through vibration, thermal, irradiation test and laboratory studies. So far, all detectors have proved their capability to withstand the severe conditions of the rocket launch. Final qualification tests on the mass and thermal model of the PAMELA apparatus are ongoing at the TsSKB-Progress factory in Samara, Russia.

Furthermore, detector performances have been verified in test experiments at the PS and SPS beams at CERN. Tests performed so far show that all the detectors of the PAMELA apparatus comply with their design performance. The latest tests were carried out in September 2003 at SPS in an almost complete flight model setup.

Integration of the flight model versions of all subdetectors is underway in Rome. PAMELA will then be taken to TsSKBProgress for integration with the Resurs DK1 satellite in early 2004. The launch is scheduled for the first half of 2004.

\section{REFERENCES}

[1] M. Simon (on behalf of the PAMELA Collaboration), "Status of the PAMELA experiment on-board of the resurs DK-1 spacecraft," in Proc. 28th Int. Cosmic Ray Conf., Tsukuba, Japan, 2003, OG 1.5, pp. 2117-2120.

[2] O. Adriani, L. Bonechi, M. Bongi, G. Castellini, R. D’Alessandro, and A. Gabbanini et al., "The magnetic spectrometer of the PAMELA satellite experiment," Nucl. Instrum. Meth., vol. A511, pp. 72-75, 2003.

[3] M. Pearce, P. Carlson, J. Lund, J. Lundquist, S. Orsi, and S. Rydstroem, "The anticounter system of the PAMELA space experiment," in Proc. 28th Int. Cosmic Ray Conf., Tsukuba, Japan, 2003, OG 1.5, pp. 2125-2128.

[4] M. Boezio, V. Bonvicini, E. Mocchiutti, P. Schiavon, G. Scian, and A. Vacchi et al., "A high granularity imaging calorimeter for cosmic-ray physics," Nucl. Instrum. Meth., vol. A487, pp. 407-422, 2002.

[5] D. Campana, G. Barbarino, M. Boscherini, W. Fuernkranz, W. Menn, and J. Mitchell et al., "The time-of-flight system of the PAMELA experiment," in Proc. 28th Int. Cosmic Ray Conf., Tsukuba, Japan, 2003, OG 1.5, pp. 2141-2144.

[6] M. Ambriola, R. Bellotti, F. Cafagna, M. Circella, C. De Marzo, and N. Giglietto et al., "PAMELA space mission: the transition radiation detector," in Proc. 28th Int. Cosmic Ray Conf., Tsukuba, Japan, 2003, OG 1.5, pp. 2121-2124.

[7] R. L. Golden, S. Horan, B. G. Mauger, G. D. Badhwar, J. L. Lacy, and S. A. Stephens et al., "Evidence for the existence of cosmic-ray antiprotons," Phys. Rev. Lett., vol. 43, pp. 1196-1199, 1979.

[8] R. L. Golden, B. G. Mauger, S. Nunn, and S. Horan, "Energy dependence of the p-bar/p ratio in cosmic rays," Astrophys. Lett., vol. 24, pp. 75-83, 1984.

[9] E. A. Bogomolov, G. I. Vasilyev, S. Y. Krut'kov, S. V. Stepanov, and M. S. Shulakova, "Antiprotons and deuterons in galactic cosmic rays," Proc. Russian Acad. Sci. (Phys. Ser.), vol. 67, pp. 447-451, 2003.

[10] R. E. Streitmatter, J. S. Stochaj, F. J. Ormes, L. R. Golden, A. S. Stephens, and T. Bowen et al., "Experimental limit on low energy antiprotons in the cosmic radiation," in Proc. 21th Int. Cosmic Ray Conf., vol. 3 (OG), Adelaide, Australia, 1990, pp. 277-280. 
[11] M. H. Salamon, S. McKee, J. A. Musser, G. Tarle, A. Tomasch, and C. R. Bower et al., "Limits on the antiproton/proton ratio in the cosmic radiation from $100 \mathrm{MeV}$ to $1580 \mathrm{MeV}$," Astrophys. J., vol. 349, pp. 78-90, 1990.

[12] M. Hof, W. Menn, C. Pfeifer, M. Simon, R. L. Golden, and S. J. Stochaj et al., "Measurement of cosmic-ray antiprotons from 3.7 to $19 \mathrm{GeV}$," Astrophys. J. Lett., vol. 467, pp. 33-36, 1996.

[13] J. W. Mitchell, L. M. Barbier, E. R. Christian, J. F. Krizmanic, K Krombel, and J. F. Ormes et al., "Measurement of $0.25-3.2 \mathrm{GeV}$ antiprotons in the cosmic radiation," Phys. Rev. Lett., vol. 76, pp. 3057-3060, 1996.

[14] A. Moiseev, K. Yoshimura, I. Ueda, K. Anraku, R. Golden, and M. Imori et al., "Cosmic-ray antiproton flux in the energy range from 200 to 600 MeV," Astrophys. J., vol. 474, pp. 479-489, 1997.

[15] M. Boezio, P. Carlson, T. Francke, N. Weber, M. Suffert, and M. Hof et al., "The cosmic ray antiproton flux between 0.62 and $3.19 \mathrm{GeV}$ measured near solar minimum activity," Astrophys. J., vol. 487, pp. 415-423, 1997.

[16] S. Orito, T. Maeno, H. Matsunaga, K. Abe, K. Anraku, and Y. Asaoka et al., "Precision measurement of cosmic-ray antiproton spectrum," Phys. Rev. Lett., vol. 84, pp. 1078-1081, 2000.

[17] M. Boezio, V. Bonvicini, P. Schiavon, A. Vacchi, N. Zampa, and D. Bergstroem et al., "The cosmic-ray antiproton flux between 3 and 49 GeV," Astrophys. J., vol. 561, pp. 787-799, 2001.

[18] A. S. Beach, J. J. Beatty, A. Bhattacharyya, C. Bower, S. Coutu, and M. A. Duvernois et al., "Measurement of the cosmic-ray antiproton-toproton abundance ratio between 4 and $50 \mathrm{GeV}$," Phys. Rev. Lett., vol. 87 , pp. 271 101/1-271 101/4, 2001

[19] Y. Asaoka, Y. Shikaze, K. Abe, K. Anraku, M. Fujikawa, and H. Fuke et al., "Measurements of cosmic-ray low-energy antiproton and proton spectra in a transient period of solar field reversal," Phys. Rev. Lett., vol. 88, pp. 051 101/1-051 101/4, 2002.

[20] A. Molnar and M. Simon, "A new calculation of the interstellar secondary cosmic ray antiprotons," in Proc. 27th Int. Cosmic Ray Conf., Hamburg, Germany, 2001, OG 043, pp. 1877-1879.

[21] J. L. Fanselow, R. C. Hartman, R. H. Hildebrand, and P. Meyer, "Charge composition and energy spectrum of primary cosmic-ray electrons," Astrophys. J., vol. 158, pp. 771-780, 1969.
[22] J. K. Daugherty, R. C. Hartman, and P. J. Schmidt, "A measuremen of cosmic-ray positron and negatron spectra between 50 and $800 \mathrm{MV}$," Astrophys. J., vol. 198, pp. 493-505, 1975.

[23] R. L. Golden, B. G. Mauger, S. Horan, S. A. Stephens, R. R. Daniel, an G. D. Badhwar et al., "Observation of cosmic ray positrons in the region from 5 to $50 \mathrm{GeV}$," Astron. \& Astrophys., vol. 188, pp. 145-154, 1987.

[24] D. Mueller and K. Tang, "Cosmic-ray positrons from 10 to $20 \mathrm{GeV}$ : a balloon-borne measurement using the geomagnetic east-west asymmetry," Astrophys. Jour., vol. 312, pp. 183-194, 1987.

[25] R. L. Golden, B. G. Mauger, S. Horan, S. A. Stephens, R. R. Daniel, and G. D. Badhwar et al., "Observations of cosmic-ray electrons and positrons using an imaging calorimeter," Astrophys. J., vol. 436, pp. 769-775, 1994

[26] R. L. Golden, S. J. Stochaj, S. A. Stephens, F. Aversa, G. Barbiellini, and M. Boezio et al., "Measurement of the positron to electron ratio in the cosmic rays above $5 \mathrm{GeV}$," Astrophys. J. Lett., vol. 457, pp. 103-106, 1996.

[27] J. M. Clem, D. P. Clements, J. Esposito, P. Evenson, D. Huber, and J. L'Heureux et al., "Solar modulation of cosmic electrons," Astrophys. J., vol. 464, pp. 507-515, 1996.

[28] S. W. Barwick, J. J. Beatty, A. Bhattacharyya, C. R. Bower, C. J. Chaput, and S. Coutu et al., "Measurements of the cosmic-ray positron fraction from 1 to $50 \mathrm{GeV}$," Astrophys. J. Lett., vol. 482, pp. 191-194, 1997.

[29] M. Boezio, P. Carlson, T. Francke, N. Weber, M. Suffert, and M. Hof et al., "The cosmic ray electron and positron spectra measured at $1 \mathrm{AU}$ during solar minimum activity," Astrophys. J., vol. 532, pp. 653-669, 2000 .

[30] M. Boezio, G. Barbiellini, V. Bonvicini, P. Schiavon, A. Vacchi, and N. Zampa et al., "Measurements of cosmic-ray electrons and positrons by the WiZard/CAPRICE collaboration," Advances Space Res., vol. 27, pp. 669-674, 2001.

[31] C. Grimani, S. A. Stephens, F. S. Cafagna, G. Basini, R. Bellotti, and M. T. Brunetti et al., "Measurement of the absolute energy spectra of cosmic-ray positrons and electrons above $7 \mathrm{GeV}$," Astron. \& Astrophys., vol. 392, pp. 287-294, 2002

[32] I. V. Moskalenko and A. W. Strong, "Production and propagation of cosmic-ray positrons and electrons," Astrophys. J., vol. 493, pp. 694-707, 1998 